\title{
The Application of Introducing Piano Accompaniment into Ruan Xian Music Teaching
}

\author{
Liao Yuning \\ Guangdong Teachers College of Foreign Language and Arts, Guangzhou, Guangdong, China
}

Keywords: piano accompaniment; Ruan Xian music; teaching

\begin{abstract}
Ruan first appeared in Qin and Han dynasty. It is a traditional Chinese national musical instrument. It was named from Ruan Xian who is one of the Seven Worthies of Bamboo Grove and plays it very well. The sound is solid, varied, and classical in appearance. In the interviews of the older generation of artists, after the founding of the People's Republic of China, it returned to the big family of traditional folk music. It is the key in the band, and the solo sound is melodious, the sound is pleasant, the background is rich in culture and history, and it is also the shining star in folk music. This paper will focus on the introduction of piano accompaniment in the Ruan Xian teaching. This paper first points out the problems in the traditional Ruan teaching and the difference between Ruan and Chinese lute playing techniques, and points out the teaching advantages according to the problems need to be paid attention in introducing the piano accompaniment into Ruan teaching. Finally, the status quo and implementation of piano accompaniment in the professional teaching of Ruan is clarified in order to better inherit and carry forward the teaching of Ruan in China.
\end{abstract}

\section{Introduction}

In order to enrich the teaching methods of Ruan music, expand its performance form and expressiveness, and improve the quality of teaching, it is an effective way to introduce piano accompaniment into the practice and teaching of Ruan music. The piano accompaniment is rich in expression techniques. It is a specialized music performing art that combines different musical forms. Therefore, many ethnic and western musical instruments are widely used in instrumental music teaching, and are used to enrich their expressiveness through piano accompaniment [1]. After years of development, how to achieve further expansion of Ruan's artistic expression and how to enrich teaching methods are problems that teachers need to think in teaching. At the same time, we can enrich the means of teaching. In the teaching and practice of Ruan music, the introduction of piano accompaniment is the embodiment of this kind of thinking.

\section{Problems in Traditional Ruan Teaching}

There are many problems in the traditional Ruan teaching, and lack of formal, rigorous and systematic training. Some students' performance postures are not standard, and the position of the hand is not correct. The artistic performance is not significant, and the rhythm is poor. It is hard to rectify the existing habit of playing. Therefore, there are many mistaken ideas about teachers due to traditional factors.

\subsection{The lack of knowledge of ancient culture, and the shallow understanding of the work}

Because of the uneven cultural level of students, there is a lack of common sense of ancient works. People's thinking will vary a lot with the gradual larger differences between ancient and modern cultural. In understanding some ancient classical music works, the students are relatively superficial, and it is difficult to start from the era and creation environment of the composer when they feel the thoughts and feelings of the works. In the face of ancient classical Chinese, students usually avoid it. It is more difficult to understand, so the performance is not satisfactory. The student's room for improvement is limited, and this problem is difficult to solve in teaching. Therefore, it is very important to improve students' knowledge of ancient cultures for their ability to 
understand musical works [2].

\subsection{The over-ambitious learning goal and the inaccurate self-positioning}

For the study of Ruan, some students blindly think that it is easier to learn before they practice it. Therefore, it is an unrealistically learning goal that is not based on their own foundation. Therefore, a chain reaction of a vicious circle is thus produced. They tried to do it in a sneak peek and pursue speed too much, so teachers are faced with great difficulties in teaching. Some students play highly difficult tracks, based on the fact that their basic skills are not solid, so the performance is not satisfactory. Teachers and parents need to pay attention to the students' self-goal and positioning.

\subsection{The passive classroom atmosphere and the traditional teaching model}

"Teaching what" and "How to teach" are the issues that teachers pay attention to in traditional classrooms. The main role of students is not fully exerted.In many cases, students who have the opportunity to perform demonstrations are poor students. Therefore, in the classroom, those students with poor foundation become the "listeners" in the classroom [3], which are closely related to the teacher's teaching mode. This kind of classroom atmosphere hinders the all-round development of poor students, and at the same time makes the classroom atmosphere more active. The teaching reform of Ruan curriculum has become imperative with the he promotion of multimedia teaching and language in the current society.

\subsection{The playing skills need to be improved and the position of holding the instrument is not standard}

In national musical instruments, it is easy to have an irregular performance posture appearing in the Ruan performance process, and it will bring problems to the spine. Because of the irregular Ruan holding posture, many students are hard to reach the requirements of playing, and they will ignore the posture of hand holding of Ruan. In order to make the performance yield twice the result with half the effort, it is necessary to master and apply the standard and correct performance posture. It is very important to improve the skill if you want the work to be touching, because it is the core of Ruan's performance. Due to the lack of proficiency in the students' playing skills, and the lack of comprehension and skill, the performance in the stage is poor and the artistic effect of the works is not ideal.

\section{The Playing Technical Differences between Ruan and Chinese Lute}

Ruan's style of performance is different from each other and has its own characteristics. And the technique is divided into finger and dialing. The technical conditions of playing are consistent with the use of natural weight, the direction of action, and the function of the main parts of the participant. The Chinese lute, which belongs to the Chinese national plucking instruments, the rolling techniques are not so different in the concept of many people. But that's not the case. The training of wrist strength is more emphasized in Ruan's primary training, and the training of finger strength is emphasized in Chinese lute. In the rolling, Chinese lute asks $2 / 3$ of the inside of the right arm to lean against the frame. Ruan asks the right elbow or the inside of the boom to lean against the frame. Compared with Chinese lute, in Ruan's rolling, the other parts of the main body of the action, as well as the joints of the index finger and the large joints of the thumb must always maintain the overall resultant force, and the right wrist should be slightly raised. Because the back shoulder is the origin of the arm strength, in the rolling action, it is necessary to pay attention to the inner smoothness of the movement and the functional coordination of the movement. Because of the high hardness and high tension of Ruan string, it is necessary to make the wrist force penetrate the fingertips to act on the string in the playing, and to realize the full application of the penetrating impact force of the wrist. At the same time, because the force is easier to transport from high to low, the finger touch point needs to be slightly lower than the upper side of the wrist. Most of the ZhongFeng touching strings are played by Chinese lute, while the thumb selects the ZhengFeng touching strings and the index finger selects the ShangPianFeng touching strings for the Ruan 
rolling [4]. Moreover, in Ruan and Chinese lute, the rolling technique belongs to the voice line technology. The lute is mainly expressed by the technique of wheel fingers, and Ruan relies on the technique of rolling. So in relative terms, Ruan uses more rolling.

\section{Problems in the Introduction of Piano Accompaniment in Ruan Professional Teaching}

\subsection{Cultivate students' ability to recognize staff}

In general, the notation is a solo spectrum of national instrumental music. However, after adding the piano accompaniment, the score chooses the form of single-line solo plus double-line large-scale piano score. When guiding students to learn, teachers need to join the piano scores from two levels, horizontally and vertically. At the same time, pay attention to their own solo spectrum. Respond to the accompaniment of the piano, the melody direction and the solo on the spectrum, especially the research, thinking and preparation in advance, the large section of the melody in the music, the coordination of the parts of the slab, etc., they need to ask piano accompaniment or professional teachers about the existed problems in time.

\subsection{Consciously train students to listen to the dynamic trend of the piano voice}

In the accompaniment, you need to actively sense the polyphony, color, melody, rhythm, harmony and other elements contained in the music. Actively exercise your own musical sensibility and comprehensive listening ability, scheduling strength and timbre changes, and constantly improve the ability to cooperate with the piano, so as to better improve learning efficiency and effectively use each accompaniment class.

\subsection{The real introduction of piano accompaniment in the Ruan professional class}

Piano accompaniment teachers need to be familiar with the instrument and have skillful playing skills. In order to carry out teaching more effectively, they need to have a good sense of cooperation. The teachers need to introduce Ruan's music performance characteristics and general situation to piano accompaniment teachers to achieve a comprehensive understanding of Ruan musical instrument. In addition, before the class, the Ruan professional teachers also need to introduce the key points, difficulties, and places of interest of the music to the piano accompaniment teachers, as well as the performance content, style characteristics, creative background, and to reach the consensus of the processing of the music[5].

\section{The Advantage of Piano Accompaniment in the Teaching of Ruan}

\subsection{Improve music literacy and professional skills}

Introducing piano accompaniment into the Ruan professional teaching, it can achieve effective training in all aspects, including speed, pitch, rhythm and so on. In most cases, especially some traditional style works are single melody lines. In terms of aesthetics, China's music is free and easy, implicit and restrained, there are a lot of music, cadenza, tail, printed word, scattered plate, etc. There are many music, usually choose to liberalize the treatment of these parts, completely rely on the player Ggood musical sensibility and superb performance. But the rhythm and speed of some of them are difficult for students to grasp. In the course of teaching, teachers choose metronome to train rhythm. But it is stiff and rigid, and it is inevitable to be mechanize. The full play of students' self-expression is not conducive to realization. Not only that, the concept of the first tone is usually chosen by the national instrumental music. It is only necessary to adjust the string relationship to play in the solo string, and the fixed pitch is consistent when tuning the string, which is often overlooked by students. It is easy to appear the problems of solo speed, intonation, rhythm and so on. Adding piano accompaniment can make them effectively trained and solved. Therefore, after learning the piano accompaniment class, the students' music literacy and professional skills have been greatly improved. 


\subsection{Enhance confidence in playing and train psychological quality}

When a student is playing solo, introducing piano accompaniment can appropriately alleviate psychological emotions such as tension. Students have not yet mastered the music to reach the proficiency stage, it is difficult to perfectly present the artistic conception of the music, and will be nervous when playing. However, when joining the piano accompaniment, the students have psychological dependence, and the two are combined into one when playing [6]. For example, in the third sections of the "Silk Road camel bell", it depicts the vast and vast desert of the western regions, and the magnificent and meandering business and travel camel teams are strong and trek, with strong infection and shock. In this double-string playing, it is required to highlight the sense of musical expression and the turbulence of the melody. It is one of the technical difficulties to maintain the strength and relaxation of the left and right hands. When playing this piece of music, many students are often difficult to balance between slack and guaranteed sound. It is easy to play the problem of dry voice and tight arms. Based on this, it is extremely important to achieve the improvement of students' double-rolling skills. And in the piano accompaniment part of this paragraph, Ruan bears the common and solo melody part. The right hand uses a chord to connect the melody, and the left hand selects the accompaniment texture of the rhythm pattern to reduce the solo pressure. The overall sound effect is more abundant, alleviating the situation that students are prone to hand stiffness. Therefore, by adding piano accompaniment, students can fully participate in music performance, eliminating psychological and physical tension in performances and training.

\subsection{Develop teaching mode}

Piano accompaniment is introduced into Ruan teaching to realize diversified artistic teaching ideas. The current "one to one" teaching mode is the choice of many instrumental music majors, which can stimulate students' enthusiasm for learning, open up a diversified teaching model, and improve the quality of teaching. The series Ruan ensemble course can be added to professional teaching to achieve vivid and all-round training of students. The piano melts harmony, music and polyphony. It is rich in texture and varied in techniques. This allows students to enjoy the piano accompaniment in the professional class, so they can feel the artistic expression and rich sound of the fusion, and experience the spark of the two different instruments in the performance of music and the sound quality [7]. Most of the teaching is the accompaniment of the piano teacher, so it can deepen the students' understanding of the music from different angles, and accept the multi-level and all-round guidance of the two teachers. Compared with the one-on-one single professional teaching mode, this can stimulate students' enthusiasm and interest in learning, while broadening the artistic vision.

\section{References}

[1] Haitian Ning. The practice of introducing Ruan music teaching into piano accompaniment [J]. The world of Music, 2016, (4):48-52.

[2] Longjian Tan. Kunming is better than the Fuchun River--The discipline construction and development of Ruan specialty of Central Conservatory of Music [J]. People's Music (Comments), 2012, (1):26-28.

[3] Haitian Ning. An analysis of the techniques and creative features of Ruan music "Yuqin Zan" [J].musical creation, 2016, (4):183-184.

[4] Jinxiu Yu. Analysis of the key points in the technical training of folk musical instrument Ruan [J]. Journal of Research on Ethnic Education, 2013, 24(4):141-144.

[5] Lixue Zhu. The application of Jinggangshan red music resources in the teaching practice of national instrumental music -- Taking Chinese Ruan as an example [J].Yellow River of the Song,2017,(19):20-21.

[6] Jinxiu Yu, Jinlong Yu. Several problems that should be paid attention to in the technical training of national musical instrument Ruan[J].Journal of Research on Ethnic Education,2011,(4):114-118.

[7] Tong Zhou. The exploration and practice of the new thinking in the teaching of Ruan [J]. Art Science and Technology, 2016, 29(1):41. 